
three-dimensional: the spacecraft will measure not just the stars' locations on the sky, but also their distances from Earth, accurate to less than 1\%. For now, the distances to only a few hundred stars are known at this level of precision.

Michael Perryman, an astronomer at the University of Bristol, UK, and former project scientist for the mission, is optimistic. "Gaia will be tremendous and transformational, a huge leap forward both in terms of the number of stars measured and the accuracy of those measurements," he says.

\section{SHELAR GARTOGRAPHY}

The keen eyesight that will make this leap possible starts with Gaia's digital camera, which uses lightgathering sensors similar to those found in consumer cameras - but 106 of them, providing a resolution of more than 900 megapixels. By contrast, the main camera on NASA's Hubble Space Telescope has two sensors with a resolution of just over 16 megapixels.

Guiding starlight into the camera are two telescopes that point $106.5^{\circ}$ apart, to take in a wide field of view. As the spacecraft spins, completing a full revolution once every 6 hours, that view will sweep across the same stars, month after month. Each star will be photographed about 70 times, producing roughly twice as much imaging data in 5 years as Hubble generated during its first 21 years in orbit.

When all the data have been analysed, they will provide a pair of coordinates for each star, pinpointing its position in the sky with an error as small as 6 microarcseconds - the size of a small coin sitting on the Moon as viewed from Earth. That is hundreds of times better than today's best catalogue, and millions of times better than the first known Western star atlas, compiled more than 2,000 years ago through naked-eye observations by the ancient Greek astronomer Hipparchus of Nicaea.

Finding a star's position in three dimensions will require further measurements. Because of a geometric phenomenon known as parallax (see 'The parallax effect'), stars appear to move from side to side as Earth orbits the Sun. The closer a star is to Earth, the larger its apparent movement, for much the same reason that trees on the side of a road seem to whiz past a speeding car, whereas a mountain in the distance barely seems to move at all. If astronomers can measure that sideto-side motion precisely, simple geometry will allow them to use the known size of Earth's orbit to calculate the star's distance.

Atmospheric turbulence so compromises such efforts that even the best modern ground-based visible-light telescopes can see parallaxes up to only about 100 parsecs (a few hundred light years). Radio telescopes are less affected, so they can see much farther - but only for objects that emit strong radio waves. From its place outside the atmosphere, Gaia, which is destined for a stable orbit that will remain fixed relative to both the Sun and Earth, should be able to obtain parallax measurements for stars up to about 10,000 parsecs away.

The same precision should let it measure a star's 'proper motion' across the sky at even greater distances. Proper motion - the result of a star's actual movement through space perpendicular to the line of sight - will show up as a steady sideways drift in the star's position,

\section{THE PARALLAX EFFECT}

As Earth travels around the

Sun, nearby stars seem

to move back and forth

relative to distant ones. Using

simple geometry, the known

size of Earth's orbit and a

measurement of the parallax

angle ( $p)$, astronomers

can determine the star's

distance (d). For an angle of

1 arcsecond, the distance is

1 parsec (3.26 light years.)

Smaller angles mean larger

distances.

Distant stars (white)
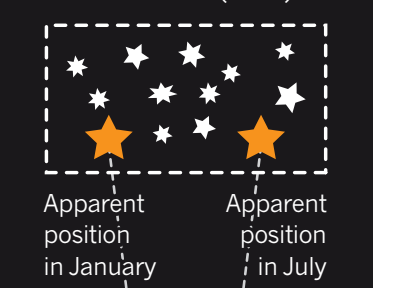

in January
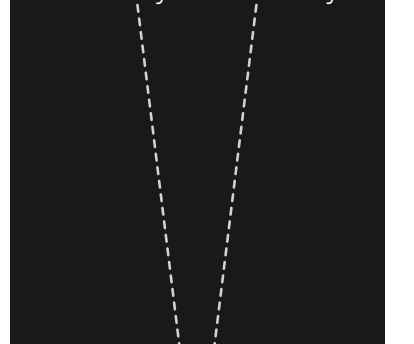

\section{.}


Gaia's limit for measuring distances with an accuracy of $10 \%$ will be 10,000 parsecs accuracy of its parallax measurements. The cuts meant that some of the problems that Gaia had intended to tackle were now out of reach. For example, the mission would no longer be able to track potentially hazardous near-Earth objects such as asteroids well enough to predict their motion for the next century - a goal that had been named a top priority by a task force led by the UK minister of science. The downgrades led Perryman to quit the project in 2006, after six years as leader of the scientific team. "I was enormously frustrated by the decision to de-scope this project, which was not made on scientific grounds," he says.

But Gaia survived and is now scheduled for launch as early as 20 November. With a bit of distance and a graveyard of space astrometry missions to reflect on, Perryman now expects big things from the mission.

\section{GAIA GOES GALAGIIC}

To start with, the cosmic census begun by Hipparcos will continue and expand. Millions of new binary stars are expected to show up, as are tens of thousands of brown dwarfs: 'failed' stars too small to ignite by fusing hydrogen. Gaia should also discover 1,000 Jupiter-sized planets - or, rather, the wobbles these objects cause in nearby stars. Closer to home, the spacecraft will get at least some data on the hundreds of thousands of Solar System asteroids expected to cross its field of view.

Where Gaia will really shine, however, will be in extending astrometry across the Milky Way (see 'Gaia's reach'). “Our unique science goal is to unravel the structure and dynamics and history of our Galaxy," says Jos de Bruijne, a systems scientist at ESA's European Space Research and Technology Centre in Noordwijk, the Netherlands, and Gaia's deputy project scientist.

Astronomers already know the basics, he says. The Milky Way is shaped something like a fried egg, with a bulge of stars in the middle surrounded by a flat stellar disk that tapers at the edges and contains the Galaxy's spiral arms. Around the disk is a diffuse sphere of old stars called the halo. But astronomers are not certain how these structures formed, or in what order (see Nature 490, 24-27; 2012). Gaia will provide one important set of clues by measuring stellar composition and brightness - data that will reveal for the first time when many stars formed, and will help astronomers to work out the ages of the Galaxy's different parts.

Another set of clues will come with Gaia's measurements of stellar movements, which astronomers can extrapolate back in time to find out how the Galaxy has evolved. Typically this is difficult because tiny errors quickly accumulate into large uncertainties. "Exactly how far back we can get is
ONATURE.COM For more about the structure and history of the Milky Way, see: go.nature.com/di7phk very much an open question," says Lindegren. But the high precision of Gaia's measurements will certainly take the extrapolation much further than before.

The measurements will also help to illuminate the many episodes of violence in the Milky Way's history. The Galaxy has grown by cannibalizing other, smaller galaxies; when they got too close, the Milky Way's gravity ripped them apart into long streams of stars that were then pulled towards the galactic centre at various angles. One such stream, torn from a dying object known as the Sagittarius dwarf galaxy billion of years ago, was found in 2002. "There are other streams out there that encode information about how the Galaxy has been developing," says Andrew Gould, an astronomer at the Ohio State University in Columbus. "Gaia will discover those streams" - and use its measurements of stellar motions to reveal how the dismemberments unfolded.

Knowing precise stellar movements should also help researchers to map out the distribution of invisible dark matter, which permeates the whole Galaxy. Dark matter emits no light, but it exerts a gravitational pull on stars, causing perturbations that should reveal themselves in Gaia's data. Those will allow astronomers to test how clumpy the dark matter is and whether it forms into disks, as theorists have proposed.

Whatever Gaia finds, one thing seems certain: its star catalogue, due to be published in 2021, will remain unsurpassed for decades. ESA is considering a planet-hunting spacecraft similar to SIM for a future mission but has yet to choose a successor to carry on Gaia's astrometric work. "We have to start thinking about it now if we want to realize something in 15 years," says Lindegren. "But we don't really know what exactly is the best way to proceed." Boosting the precision significantly would be an enormous technological challenge. An easier path would be to fly another Gaia mission with the same specifications in 20 years, after the stars have moved noticeably, to better pin down their positions and velocities.

Another proposed follow-on mission would examine parts of the Milky Way to which Gaia will be blind. Dust will obscure the Galaxy's bulge and some far-away parts of its disk from Gaia's visible-light gaze - but would pose no problem to an instrument looking for infrared radiation.

Or perhaps Gaia itself will upend the whole discussion. As astrometry sharpens its focus, there is always the exciting possibility that something wholly unexpected could be found. "Science often progresses by making detailed measurements," says Kulkarni. "Sometimes you see a deviation — something that turns out to be profound."

Devin Powell is a freelance writer currently based in Singapore. 